\title{
Visualisation du mélange gazeux au sein de la chambre de combustion des moteurs par la fluorescence induite par laser
}

Les contraintes environnementales et réglementaires de plus en plus strictes imposent le développement de nouveaux moteurs à combustion interne pour le secteur du transport routiel. Les évolutions législatives visent à réduire les émissions polluantes telles que les oxydes d'azote (NOx), le monoxyde de carbone (CO) et les hydrocarbures imbrûlés $(\mathrm{HC})$ d'une part, et à limiter les émissions de dioxyde de carbone $\left(\mathrm{CO}_{2}\right)$ issues de la combustion des hydrocarbures d'origine fossile d'autre part. Ce dernier point exige une amélioration de l'efficacité énergetique des moteurs.

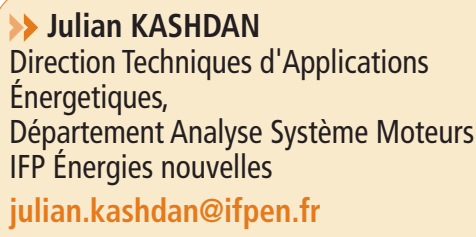

\section{Nouveaux modes de combustion}

Des systèmes de combustion innovants permettant de répondre à ces contraintes sont en cours de développement. Par exemple, la réduction de la cylindrée unitaire des moteurs essence, compensée par un système de suralimentation adapté, est une voie éprouvée pour l'augmentation du rendement moyen des moteurs et donc la réduction des émissions de $\mathrm{CO}_{2}$. La combustion diluée, par l'air ou par des gaz d'échappement recirculés est aussi une voie en cours d'exploration par les constructeurs d'automobiles. Ce type de combustion, basée sur la mise en œuvre de forts taux d'EGR (recyclage des gaz d'échappement) ou d'un mélange pauvre, peut être mis en œuvre dans les moteurs à allumage commandé pour améliorer leur rendement. La combustion essence stratifiée est un levier additionnel permettant d'envisager des forts taux d'EGR jusqu'aux très faibles charges, tout en maximisant le rendement énergétique. Cependant, la stabilité d'initiation de la flamme est un facteur limitant pour ces modes de combustion, réduisant fortement le gain $\mathrm{CO}_{2}$ atteignable.
En motorisation diesel, le challenge reste la réduction des émissions de NOx et de particules. Depuis de nombreuses années, une stratégie de combustion diesel homogène basée sur I'utilisation de très forts taux $d^{\prime} E G R$, une réduction du taux de compression et la formation de mélanges air/carburant relativement homogènes a été étudiée et développée. Ce mode de combustion dit « combustion basse température » permet de limiter les émissions de NOx sans avoir recours à un système de post-traitement dédié.

Dans le processus de développement de ces nouveaux modes de combustion, la préparation du mélange carburant/air est un paramètre clef. En effet, le dégré d'homogénéité/hétérogénéité du mélange influence fortement l'initiation et le déroulement de la combustion dans les moteurs essence comme dans les moteurs Diesel. Les paramètres qui pilotent la distribution et la qualité du mélange doivent être optimisés afin d'améliorer la combustion et en conséquence limiter les niveaux d'émissions polluantes. Pour cela, il est nécessaire d'avoir recours à des techniques de visualisation qui permettent de caractériser spatialement et quantitativement le mélange au sein de la chambre de combustion.

\section{Les techniques d'imagerie et de mesures optiques appliquées aux moteurs}

Les techniques de visualisation et de mesures non-intrusives dites « diagnos- tics optiques» sont appliquées dans le milieu de la recherche et développement des moteurs à combustion interne depuis plus de 20 ans. Ces techniques de diagnostics optiques permettent d'obtenir des informations spatiales et temporelles sur les phénomènes qui régissent la préparation du mélange, la combustion ainsi que la formation d'émissions polluantes au sein de la chambre de combustion. L'utilisation combinée de caméras et de lasers spécialement conçus pour des activités de recherche permet d'accéder à de nombreuses grandeurs physiques telles que la concentration des espèces, la vitesse des écoulements gazeux et la température au sein de la chambre de combustion d'un moteur. L'évolution des niveaux d'énergie des lasers, la sensibilité et la vitesse des caméras ont permis une progression non interrompue de ces techniques de diagnostics en termes de précision et de grandeurs mesurées. Enfin, les durées de pulse très courtes des lasers utilisés ( 5 à $10 \mathrm{ns)} \mathrm{offrent} \mathrm{la} \mathrm{capa-}$ cité de réaliser des mesures résolues temporellement au cours du cycle moteur.

\section{La visualisation du mélange par fluorescence induite par laser}

Une des techniques les plus utilisées est la technique de fluorescence induite par laser qui est particulièrement adaptée à la mesure de la concentration d'une espèce et de la température du mélange gazeux. 


\section{ㅁiagnastics aptiques et autamabile / CAHIER TECHNIDUE 35}

Cette technique permet d'obtenir des visualisations bidimensionnelles grâce à un éclairage de la zone de mesure par plan laser. Le signal de fluorescence est ensuite observé par une caméra CCD. En général, les caméras $C C D$ sont équipées d'intensificateurs qui permettent d'améliorer significativement le rapport signal sur bruit de l'image. Une caméra intensifiée offre de plus la capacité de contrôler très précisément le temps d'exposition (environ quelques ns) permettant ainsi de limiter la collection d'émissions lumineuses parasites.

Cette technique est basée sur la collection de lumière émise par une molécule dite "traceur» suite à une excitation laser accordée sur une longueur d'onde d'absorption de cette molécule. Sous certaines conditions de maîtrise du dispositif expérimental, le signal de fluorescence récolté peut être proportionnel au nombre de molécules excitées, voire à la concentration de la molécule traceur après corrections des effets de la température et de désexcitations parasites (02).

Plusieurs grandeurs physiques impactant directement le moteur et son niveau d'émissions polluantes peuvent être mesurées par la technique de fluorescence induite par laser : la concentration du carburant, la richesse (ratio air/fuel), la température, la composition du carburant ainsi que la concentration des gaz résiduels. Afin de visualiser la formation du mélange carburant/air, il est souvent nécessaire de rajouter un traceur fluorescent au carburant. Idéalement, le comportement du traceur sélectionné doit être identique au carburant en termes de caractéristiques d'évaporation, de diffusivité et de réactivité (auto-inflammation et vitesse de combustion). Cependant, il est très difficile de trouver un traceur qui remplit l'ensemble de ces critères.

L'application des techniques de diagnostics optiques telles que la fluorescence induite par laser nécessite l'utilisation de moteurs à accès optiques dits «transparents». Ces moteurs de recherche offrent un ou plusieurs accès optiques à la chambre de combustion spéci- fiquement implantés lors de la conception du moteur. Les cylindres et les pistons sont partiellement ou totalement usinés dans un matériau transparent (saphir ou en quartz). La figure 2 montre une photo d'un moteur monocylindre optique à I'IFP Énergies nouvelles utilisé pour l'application de techniques de diagnostics optiques. La figure 2 montre le montage expérimental correspondant à la technique de fluorescence induite par laser appliquée sur moteur optique.

\section{Visualisation de la distribution du mélange sur un moteur essence à injection directe}

Une séquence d'images de la distribution du mélange au sein de la chambre de combustion d'un moteur IDE (Injection Directe Essence) est montrée figure 3. Ces images ont été obtenues grâce à la technique de fluorescence induite par laser. Dans cette étude, un carburant mono-composant (iso-octane) dont la température d'ébullition est comparable à celle de l'essence commerciale, a été tracé avec un couple de traceurs TEA/ benzène. L'excitation de la fluorescence est réalisée grâce à un plan laser 2D issu d'un laser Nd:YAG laser quadruplé en fréquence (longueur d'onde d'excitation de $266 \mathrm{~nm}$ ). Les images présentées correspondent à un plan vertical coïncidant avec l'axe de symétrie de la chambre de combustion. Le signal de fluorescence a été détecté par une caméra intensifiée 16 bits (Princeton Instruments PIMax).

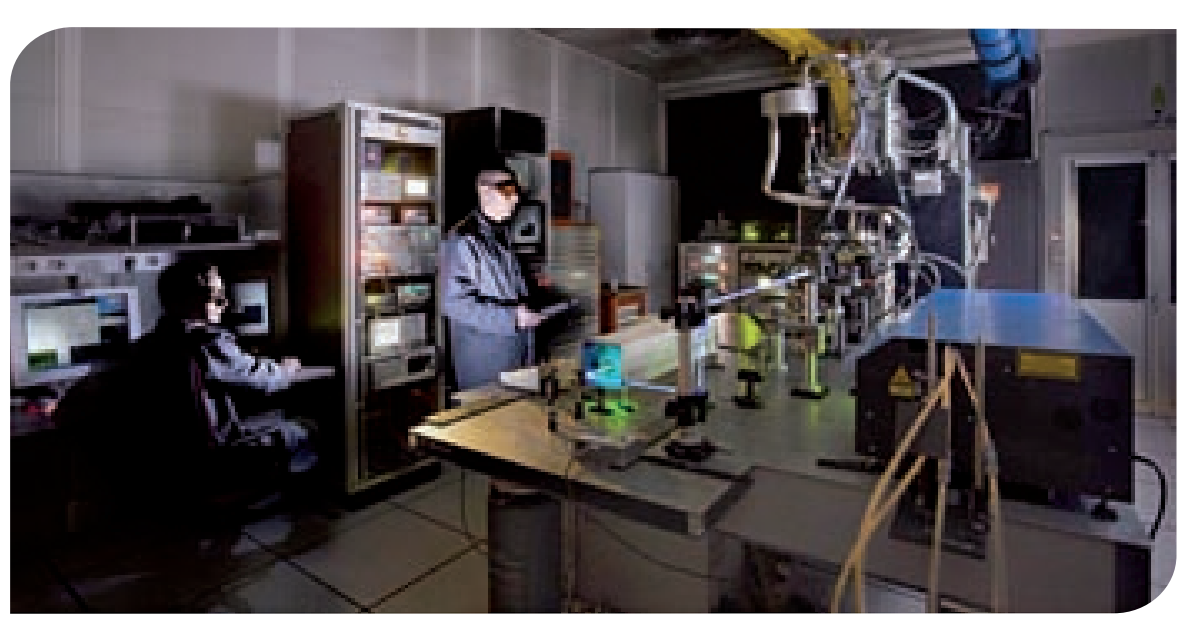

Figure 2. Photo montrant le dispositif expérimental d'une technique de visualisation par diagnostics optiques appliquée sur un moteur transparent à I'IFP Énergies nouvelles. 


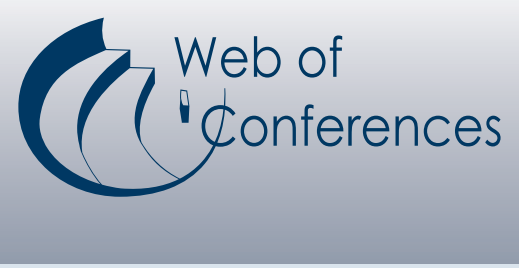

\section{Annoncez gratuitement vos prochaines \\ manifestations sur}

www.webofconferences.org

\section{Agenda international de conférences \\ Recherchez un congrès ou une conférence \\ Annoncez gratuitement une manifestation tion interne. \\ Visualisation de l'injection sur un moteur Diesel à injection directe}

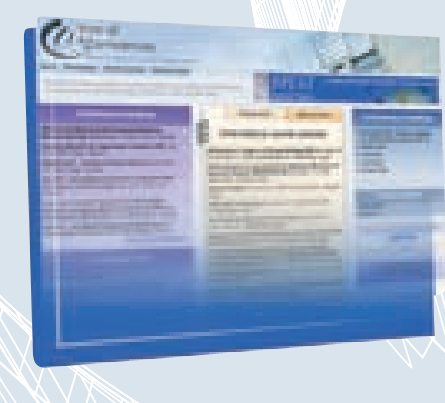

Publication et diffusion

d'actes de conférence

Publication en ligne sur

un site web dédié

Libre accès à tous les

documents

Articles identifiables et

citables (DOI, Crossref)

Hébergement sans limite

de temps

Création de collections

Impression de livres

et/ou de CDs d'impact du carburant sur les parois de la chambre. Ces informations peuvent nous servir à la fois pour (1) améliorer notre compréhension des phénomènes physiques et (2) valider les approches de simulations numériques qui sont de plus en plus utilisées dans le processus de développement des moteurs à combus-

La figure 4 montre une séquence d'images de l'injection du carburant dans un moteur Diesel obtenue avec la technique de fluorescence induite par laser. Le traceur fluorescent naphthalène a éte rajouté à un carburant mono-composant (dodécane) qui est représentatif des composants légers du gasoil commercial. Les visualisations permettent $d$ 'identifier les six jets du carburant qui pénètrent dans la chambre de combustion, impactant éventuellement les parois. Il est possible de quantifier la dispersion jet à jet ainsi que de mesurer la pénétration des phases liquide et vapeur du carburant. Le choix du système d'injection, les caractéristiques géométriques de l'injecteur ainsi que les conditions de fonctionnement
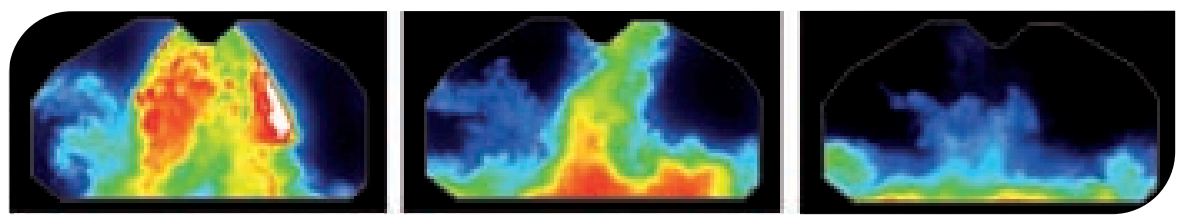

Figure 3. Séquence temporelle de 3 images montrant l'injection du carburant au sein de la chambre de combustion d'un moteur allumage commandé à injection directe essence (IDE). La technique de fluorescence induite par laser a été utilisée pour acquérir ces images.

peuvent influencer fortement la répartition du carburant au sein de la chambre. Des études expérimentales détaillées sont alors réalisées et les résultats obtenus permettent d'orienter les choix de définition technique de la chambre de combustion ; aspects primordiaux dans le développement et l'optimisation des systèmes de combustion pour atteindre les objectifs visés dans le contexte d'une réduction d'émissions polluantes et émissions de $\mathrm{CO}_{2}$.

\section{Références}

Schulz, C., Sick, V., «Tracer-LIF diagnostics : quantitative measurement of fuel concentration, temperature and fuel/air ratio in practical combustion systems», Prog. in Energy \& Comb. Sci. 31 (2005), 75-121.

Kashdan, J. T., Anselmi, P., Walter, B., «Advanced injection strategies for controlling low-temperature diesel combustion and emissions», SAE Transactions, Int. J. Engines, 2(1): 1835-1872, 2009.

Kashdan, J. T., Mendez, S., Bruneaux, G., «On the origin of unburned hydrocarbon emissions in a wall-guided, low Nox Diesel combustion system» SAE Transactions, Journal Fuels \& Lubricants, 4(116): 234-257.

Kashdan, J.T., Cherel, J., Thirouard, B., «A Study of Combustion Structure \& Implications on Post-0xidation Under Homogeneous \& Stratified Operation in a DISI Engine», SAE Transactions, Int. Journal Engines, SP2016.

Kashdan, J. T., «Tracer LIF Visualisation Studies of Piston-Top Fuel Films in a Wall-Guided, Low NOx Diesel Engine», SAE paper 2008-01-2474, 2008.
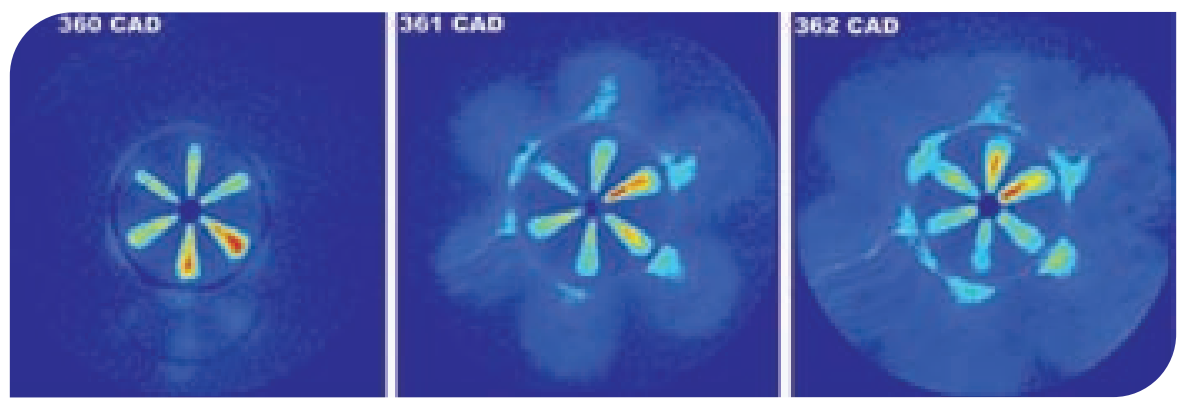

Figure 4. Séquence temporelle de 3 images montrant l'injection du carburant au sein de la chambre de combustion d'un moteur Diesel à injection directe (DID). La technique de fluorescence induite par laser a été utilisée pour acquérir ces images. 